\title{
NUEVAS ASOCIACIONES DE ESPECIES DE PARASITOIDES Y ANASTREPHA DISTINCTA (DIPTERA: TEPHRITIDAE) EN EL SOCONUSCO, CHIAPAS, MÉXICO
}

Ruiz, L.C., A. Oropeza \& J. Toledo. 2011. New associations of parasitoids species and Anastrepha distincta (Diptera: Tephritidae) in the Soconusco, Chiapas, Mexico. Acta Zoológica Mexicana (n. s.), 27(3): 813-818.

ABSTRACT. We report five native species of parasitoids as new associations with larvae of $A$. distincta, obtained from fruits of Inga spuria, I. laurina and I. micheliana.

Anastrepha distincta Greene es una especie de tefrítido del grupo fraterculus de poca importancia agrícola en México. Su distribución en el Continente Americano es amplia debido principalmente a que la distribución del género Inga, que es su principal hospedero, también es extensa (Hernández-Ortiz 1992, Sousa 1993). Los árboles del género Inga son ampliamente usados como sombra del cultivo de café (Coffea arabica L.); además, son comunes en selvas altas subdeciduas de la región Neotropical (Miranda 1998).

Las investigaciones realizadas en diferentes regiones tropicales de América han reportado solo tres especies de parasitoide atacando larvas de $A$. distincta (Jiron \& Mexzón 1987, Canal-Daza et al. 1994, Katiyar et al. 1995). En la región del Soconusco, Chiapas, México, utilizando muestreos dirigidos, se encontraron tres especies de parasitoides en éste tefrítido asociados a dos especies de Inga (Oropeza et al. 2008). Debido al amplio uso de árboles del género Inga en dicha región y a la prevalencia de condiciones ambientales propicias, la población de $A$. distincta es abundante (Celedonio-Hurtado et al. 1995, Malo et al. 1987). La importancia de identificar especies de parasitoides en estos frutos radica en que estos últimos juegan un papel como reservorio de tefrítidos de interés agrícola (Ovruski et al. 2000). En ocasiones estos reservorios naturales pueden contribuir a mantener las poblaciones de parasitoides en momentos críticos y tener un impacto en la dinámica poblacional de la plaga.

En el presente trabajo se reportan nuevas asociaciones de especies de parasitoides con larvas de $A$. distincta, colectadas en 2008 y 2009 durante el periodo de fructifi- 
cación de Inga spuria H. \& B. (ene-mar), I. laurina Wild (feb-abr), y I. micheliana Harms (mar-abr) en el Soconusco, Chiapas.

Las condiciones topográficas y ambientales del sitio de estudio son descritas en Oropeza et al. (2008). Los frutos colectados fueron trasladados al laboratorio de moscas de la fruta de El Colegio de la Frontera Sur, Unidad Tapachula, Chiapas, en donde fueron contados y pesados en una balanza granataria (Ohaus, Pine Brook, NJ); después fueron colocados en charolas de plástico $(55 \mathrm{~cm} \times 45 \mathrm{~cm} \times 12 \mathrm{~cm})$ en donde permanecieron entre cinco y ocho días bajo condiciones ambientales de $26 \pm 1{ }^{\circ} \mathrm{C}, 75 \pm$ 5\% de H.R. y un fotoperiodo de 12:12 luz:oscuridad, hasta que las larvas alcanzaron el tercer estadio. Posteriormente los frutos fueron abiertos para extraer las larvas, las cuales fueron colocadas en contenedores de plástico (capacidad para $500 \mathrm{~mL}, 10 \mathrm{~cm}$ de diámetro $\times 12 \mathrm{~cm}$ de alto) con vermiculita húmeda para promover la pupación. Las pupas permanecieron en los contenedores por 13 días (a las condiciones ambientales indicadas previamente) y después fueron separadas de la vermiculita con un tamiz (Malla 18). Las pupas fueron mantenidas en contenedores cubiertos con malla fina (tela tull, con una abertura de $0.5 \mathrm{~mm}$ ) hasta que emergieron los adultos de moscas y/o parasitoides. Los adultos de $A$. distincta fueron identificados usando claves taxonómicas por Hernández-Ortiz (1992). Los parasitoides emergidos fueron separados por sexo, cuantificados y posteriormente colocados en frascos entomológicos con alcohol al 70\%. La determinación de las especies se hizo con base en las características morfológicas descritas en claves dicotómicas (Ovruski et al. 1996, Wharton \& Marsh 1978, Wharton \& Gilstrap 1983).

Un total de $137.23 \mathrm{~kg}$ de frutos de los tres hospederos (Inga spuria, I. laurina y I. micheliana) fueron colectados de los cuales se recuperó un total de 9,622 larvas de $A$. distincta de donde se obtuvieron 96 parasitoides en fase adulta (Cuadro 1). Los parasitoides fueron clasificados en cinco especies, y ocho ejemplares solo fueron identificados a nivel de género. En este estudio, Odontosema anastrephae Borgmeier fue la especie más abundante, seguida de Aceratoneuromyia indica Silvestri y Doryctobracon areolatus Szépligeti fue la especie menos representada con solo 3 individuos (Cuadro 2). I. micheliana con 40.8 larvas $/ \mathrm{kg}$ de fruta fue el hospedero con menor grado de infestación de $A$. distincta. El caspirol (I. laurina) registró una infestación intermedia con 66.8 larvas $/ \mathrm{kg}$ de fruta pero no se obtuvieron parasitoides. En el cuajinicuil (I. spuria) se registró una infestación promedio de 72.8 larvas/kg de fruta. En este caso se obtuvo la mayor cantidad y diversidad de parasitoides durante 2008 (Cuadro 1). En general, se puede considerar que el grado de parasitismo registrado en larvas de $A$. distincta obtenidas de las tres especies de frutos hospederos fue bajo $(\sim 1.0 \%)$.

A pesar de que $A$. distincta es abundante en la región neotropical, se han reportado pocas especies que la parasitan. En Venezuela es parasitada por Aganaspis pelleranoi (Brèthes) (Katiyar et al. 1995), en Costa Rica por D. areolatus (Jiron \& Mexzón 


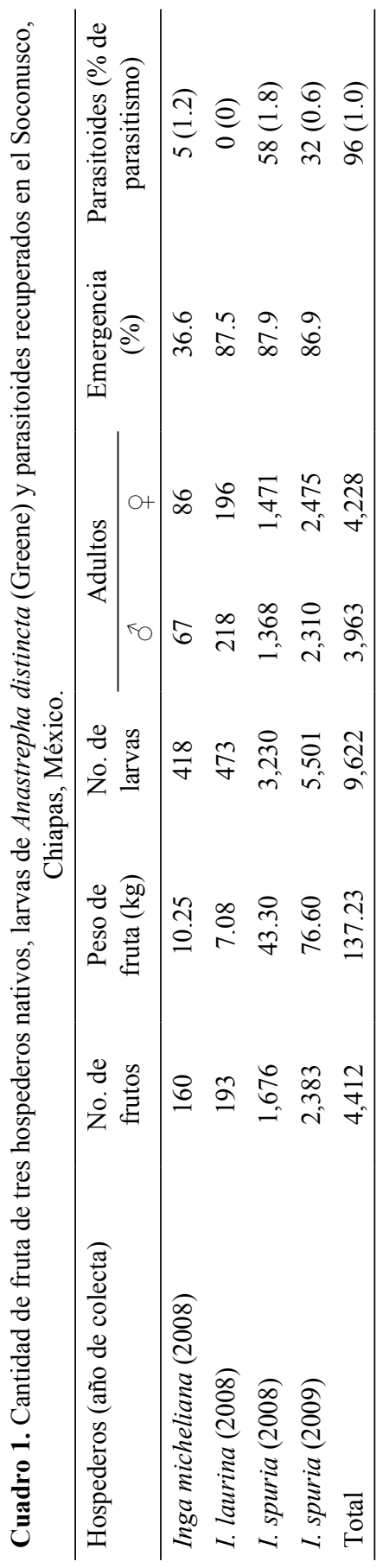


Ruiz et al.: Asociaciones entre parasitoides y Anastrepha distincta

Cuadro 2. Parasitoides de larvas de Anastrepha distincta (Greene) obtenidos de tres especies de frutos hospederos colectados en el Soconusco, Chiapas, México.

\begin{tabular}{lcccccc}
\hline \multicolumn{1}{c}{ Especies } & \multicolumn{2}{c}{2008} & \multicolumn{2}{c}{2009} & \multicolumn{2}{c}{ Total } \\
\cline { 2 - 7 } & + & 0 & + & 0 & + & ^ \\
\hline Aceratoneuromya indica & 0 & 0 & 17 & 2 & 17 & 2 \\
Doryctobracon crawfordi & 5 & 4 & 4 & 0 & 9 & 4 \\
D. areolatus & 0 & 0 & 2 & 1 & 2 & 1 \\
Doryctobracon sp. & 5 & 1 & 0 & 0 & 5 & 1 \\
Odontosema anastrephae & 21 & 25 & 0 & 0 & 21 & 25 \\
Opius hirtus & 1 & 0 & 5 & 1 & 6 & 1 \\
Utetes sp. & 2 & 0 & 0 & 0 & 2 & 0 \\
Total & 34 & 30 & 28 & 4 & 62 & 34 \\
\hline
\end{tabular}

1987) y en la región del Amazonas, Brazil, por especies del género Opius sp. (CanalDaza et al. 1994). Recientemente se reportó que en el Soconusco, Chiapas, México, A. distincta es parasitada por dos especies introducidas y una especie nativa (Oropeza et al. 2008). En este trabajo se reportan cinco especies más (todas nativas) de parasitoides asociadas a larvas de esta especie de mosca de la fruta, lo que da un total de ocho especies que la parasitan en condiciones naturales.

El bajo porcentaje de parasitismo es muy común en diferentes hospederos de Anastrepha. Factores como el refugio que ofrecen los hospederos y las condiciones climáticas entre otros, limitan la acción de los parasitoides. En este trabajo al parecer las condiciones climáticas presentes durante el periodo de muestreo no fueron las ideales para que el parasitismo fuera favorecido. En este sentido, González-Hernández y Tejada (1979) y Sivinski et al. (2000) reportaron que el grado de parasitismo de la especie nativa Doryctobracon crawfordi (Viereck) fue mayor y más frecuente cuando la humedad y la temperatura contribuyeron en forma favorable para que se diera dicha interacción, además de que la disponibilidad del hospedero se incrementó.

Inga spuria es una especie que tiene dos periodos de fructificación al año (Sousa 1993), es el hospedero que ha registrado mayor infestación por A. distincta y las larvas son parasitadas por un mayor número de especies de parasitoides (Oropeza et al. 2008). Sin embargo, en el año en que se hizo este estudio esta planta no fructificó durante el período de lluvias, por lo que no se hizo una comparación de la diversidad y abundancia de parasitoides entre ambas épocas. A pesar de que el parasitismo natural fue bajo, pero si consideramos el número de especies de parasitoides asociadas a $A$. distincta, se confirmó que el género Inga es un reservorio de parasitoides nativos, y juega un papel crucial cuando los parasitoides no encuentran hospederos para mantener su población. Este hecho sugiere que se debe analizar el manejo de estos hábitats 
como importantes sitios de refugio para que el control biológico de moscas de la fruta tenga mayor sostenibilidad. Sobre todo porque algunas de las especies de parasitoides reportadas aquí, tienen potencial (pej. D. crawfordi) para el control de poblaciones de tefrítidos que son plagas.

\section{LITERATURA CITADA}

Canal-Daza, N. A., R. A. Zucchi, N. M. Da Silva \& F. L. Leonel, Jr. 1994. Reconocimiento de las especies de parasitoides (Hym.: Braconidae) de moscas de las frutas (Dip.: Tephritidae) en dos municipios del Estado de Amazonas, Brasil. Boletín del Museo de Entomología de la Universidad del Valle, 2: 1-17.

Celedonio-Hurtado, H., M. Aluja \& P. Liedo. 1995. Adult population fluctuations of Anastrepha species (Diptera: Tephritidae) in tropical orchard habitats of Chiapas, México. Environmental Entomology, 24: 861-869.

González-Hernández, A. \& L. O. Tejada. 1979. Fluctuación de la población de Anastrepha ludens (Loew) y de sus enemigos naturales en Sargentia greggii S. Watts. Folia Entomológica Mexicana, 41: 49-60.

Hernández-Ortiz, V. 1992. El género Anastrepha Schiner en México (Diptera: Tephritidae). Taxonomía, distribución y sus plantas huéspedes. Instituto de Ecología y Sociedad Mexicana de Entomología. Xalapa, Veracruz, México. 162 pp.

Jiron, L. F. \& R. G. Mexzon. 1987. Parasitoid Hymenopterans of Costa Rica: Geographical distribution of the species associated with fruit flies (Diptera: Tephritidae). Entomophaga, 33: 79-86.

Katiyar, K. P., J. Camacho, F. Geraud \& R. Matheus. 1995. Parasitoides hymenópteros de moscas de las frutas (Diptera: Tephritidae) en la región occidental de Venezuela. Revista de la Facultad de Agronomía, 12: 303-312.

Malo, E., P.S. Baker \& J. Valenzuela. 1987. The abundance of species of Anastrepha (Diptera: Tephritidae) in the coffee producing area of coastal Chiapas, Southern Mexico. Folia Entomológica Mexicana, 73: 125-140.

Miranda, F. 1998. La Vegetación de Chiapas. Edit. Talleres Gráficos del Estado de Chiapas. 3a. Edic. Tuxtla Gutiérrez, Chiapas. México. 596 pp.

Oropeza, A., L. C. Ruiz \& J. Toledo. 2008. Larval parasitoids associated to Anastrepha distincta (Diptera: Tephritidae) in two host fruits at the Soconusco Region, Chiapas, Mexico. Florida Entomologist, 91: 498-500.

Ovruski, S. M., S. Fuentes., F. Núñez \& J. G. Granados-Zuñiga. 1996. Himenópteros "parasitoides de moscas de la fruta" (Diptera: Tephritidae) presentes en la República de El Salvador. Revista de la Sociedad de Ingenieros Agrónomos de El Salvador, 8: 8-14.

Ovruski, S., M. Aluja, J. Sivinski \& R. Wharton. 2000. Hymenopteran parasitoids on fruit-infesting Tephritidae (Diptera) in Latin America and the southern United States: Diversity, distribution, taxonomic status and their use in fruit fly biological control. Integrated Pest Management Reviews, 5: $1-107$.

Sivinski, J., J. Piñero \& M. Aluja. 2000. The distributions of parasitoids (Hymenoptera) of Anastrepha fruit flies (Diptera: Tephritidae) along an altitudinal gradient in Veracruz, México. Biological Control, 18: 258-269.

Sousa, M. 1993. El género Inga (Leguminosae: Mimosoideae) del sur de México y Centroamérica, estudio previo para la flora Mesoamericana. Annals of the Missouri Botanical Garden, 80: 223-269.

Wharton, R.A. \& P. M. Marsh. 1978. New world Opiinae (Hymenoptera: Braconidae) parasitic on Tephritidae (Diptera). Journal of the Washington Academy of Sciences, 68: 147-167. 
Ruiz et al.: Asociaciones entre parasitoides y Anastrepha distincta

Wharton, R. A. \& F. E. Gilstrap. 1983. Key to and status of opiine Braconid (Hymenoptera) parasitoids used in biological control of Ceratitis and Dacus (Diptera: Tephritidae). Annals of the Entomological Society of America, 76: 721-742.

AGRADECIMIENTOS. A Juan Rull y a dos revisores anónimos por sus valiosas sugerencias que contribuyeron a mejorar la calidad del trabajo. A Gustavo Rodas y Ezequiel de León (ECOSUR), por el apoyo en la colecta y manejo de la fruta.

\author{
Lía C. Ruiz ${ }^{1}$, Azucena Oropeza ${ }^{2}$ \& Jorge Toledo ${ }^{2}$ \\ ${ }^{1}$ Programa Operativo MOSCAFRUT (SAGARPA), \\ Av. Central Poniente 14, Tapachula, Chiapas 30700, México. \\ ${ }^{2}$ Departamento de Entomología Tropical. \\ El Colegio de la Frontera Sur. \\ 30700 Tapachula, Chiapas, México. \\ Autor para correspondencia. < jtoledo@ecosur.mx>
}

